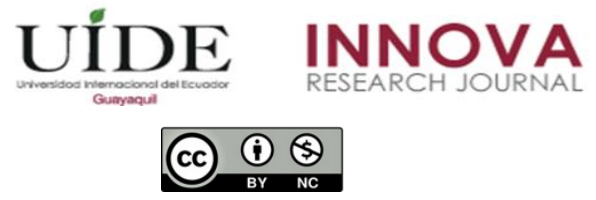

INNOVA Research Journal, ISSN 2477-9024

(Enero-Abril 2021). Vol. 6, No.1 pp. 177-194

DOI: https://doi.org/10.33890/innova.v6.n1.2021.1472

URL: http://revistas.uide.edu.ec/index.php/innova/index

Correo: innova@uide.edu.ec

\title{
Rotación del personal de nivel jerárquico superior y el clima laboral en el Servicio de Contratación de Obras SECOB
}

\section{Rotation of higher-level personnel and the work environment in the SECOB Works Contracting Service}

\author{
Diana Verónica Parra Vaca \\ https://orcid.org/0000-0002-5210-4827 \\ Universidad Técnica de Ambato, Ecuador
}

Autor para correspondencia: diana.parravaca@gmail.com

Fecha de recepción: 20 de julio de 2020 - Fecha de aceptación: 16 de noviembre de 2020

\section{Resumen}

El presente trabajo de investigación tiene como objetivo comprobar la incidencia de la rotación del personal de nivel jerárquico superior en el clima laboral de una institución del sector público, específicamente en el Servicio de Contratación de Obras SECOB; para la medición de cada una de las variables se aplicó un instrumento utilizado por varios autores en temas de clima laboral, el mismo que fue validado a través del método juicio de expertos; así mismo, la confiabilidad se obtuvo a través del coeficiente Alfa de Cronbach, la encuesta fue aplicada a una muestra de 163 funcionarios de la institución, en los cuales se midieron las dimensiones: comunicación interna, reconocimiento, relaciones interpersonales en el trabajo, toma de decisiones, entorno físico, compromiso y adaptación al cambio, consideradas dentro de la variable clima laboral, y por otro lado para la variable rotación de personal de nivel jerárquico superior se consultó si existió rotación y el número de veces que rotaron los directivos en cada una de las áreas durante el año 2018, año escogido para la investigación por presentar el mayor número de cambios de jefes en la institución. La investigación realizada permitió comprobar que sí existe incidencia entre las variables estudiadas en la institución mencionada, pese a ello, se identificaron varias limitaciones en el desarrollo de la misma, al no existir suficiente literatura que relacione dichos constructos, tanto en el sector público como en el sector privado, siendo estos temas de relevancia para el mejoramiento del clima laboral.

Palabras claves: motivación laboral; motivación intrínseca; motivación extrínseca; flexibilidad de horario; fomento de la investigación.

\footnotetext{
Abstract

The purpose of this research work is to verify the incidence of the rotation of higher-level personnel in the work environment of a public sector institution, specifically in the Servicio de Contratación de Obras SECOB; to measure each variable, an instrument used by several authors in matters of work climate was applied, which was validated through the method of expert judgment; likewise, reliability was obtained through Cronbach's alpha coefficient, the survey was applied to a sample
} 
of 163 institution officials, the dimensions that were measured: internal communication, recognition, interpersonal relationships at work, taking Decisions, physical environment, commitment and adaptation to change, considered within the variable work environment, on the other hand, for the variable rotation of higher-level personnel, it was consulted whether there was rotation and the number of times managers rotated in each area during 2018, the year chosen for the research for presenting the greatest number of changes of heads in the institution. The research carried out made it possible to verify that there is an incidence among the variables studied in the aforementioned institution, despite this, several limitations were identified in its development, as there is not enough literature that relates these constructs, both in the public sector and in the private sector, being these issues of relevance for the improvement of the working environment

Keywords: organizational climate; management turnover; executive staff; government sector; organizational development.

\section{Introducción}

La presente investigación pretende estudiar la relación que pueda existir entre los constructos Rotación de personal de Nivel Jerárquico Superior y el Clima laboral, es decir como las variables se relacionan e influyen la una sobre la otra, para este fin, se han revisado y analizado diferentes trabajos y estudios realizados sobre estas variables, tanto a nivel nacional como internacional.

A nivel internacional, con respecto a la variable rotación de personal de nivel jerárquico superior, (Ysa y Salvador, 2015) en su trabajo Liderazgo y dirección pública: el ciclo de vida del cargo directivo, abordan el análisis de la carrera horizontal del directivo público a lo largo de su mandato, frente a una aproximación tradicional, vertical, de análisis de promoción en carrera. En el estudio indican que el directivo público es actualmente una pieza clave para la toma de decisiones, así como para el diseño e implementación de políticas públicas, a través de sus acciones influye en la consecución de objetivos (Vermeeren, Kuipers, y Steijn, 2014) y es clave para superar problemas inherentes al sector público (Behn, 1998) (Fairholm, 2004).

De acuerdo con lo antes indicado, en este estudio se resalta la importancia que tiene el Directivo Público en la consecución de objetivos de la organización, alineados a las políticas públicas del gobierno nacional, ya que a través de sus decisiones y acciones conduce a la organización al cumplimiento de las actividades que permitan alcanzar la misión institucional.

Así mismo, en dicho estudio se cita a (Eitzen y Yetman, 1972), los cuales afirman que existe una relación curvilínea entre la duración de un mandato de un entrenador y la efectividad de un equipo de baloncesto: a mayor duración en el cargo, mejores resultados del equipo, hasta un determinado momento, en el cual los resultados empiezan a disminuir. Las autoras incluyen el concepto de ciclo de vida, entendido este como una serie de etapas por las que puede pasar un directivo desde la aceptación del cargo hasta su terminación, este concepto es importante porque permite identificar en las diferentes etapas, los cambios que tienen influencia en los resultados de la organización. 
En el estudio mencionado se establece la importancia de la duración en el cargo y se define el ciclo de vida, con lo cual en relación a la presente investigación se afirma que mientras más tiempo dure una persona en un cargo, mejores resultados se obtendrán, por lo que en el sector público se debería procurar la estabilidad de los cargos de nivel jerárquico superior y reducir de acuerdo al ciclo de vida normal la rotación de este tipo de personal, pues de esta forma se podrá dar continuidad y consistencia a los procesos y actividades de la gestión pública que permitan mejorar el servicio público y alcanzar los objetivos institucionales.

Finalmente, en su investigación concluyen que la duración del mandato puede asociarse a buenos o malos resultados, pero en distintas etapas del ciclo de vida, así mismo, el modelo del ciclo de vida del mandato del directivo público tiene implicaciones significativas para la eficacia organizativa, en el caso de directivos públicos cuyos mandatos pueden ser muy cortos (por ejemplo, solo una o dos etapas de las cinco), se puede esperar eficacias menores, por lo cual las organizaciones deben estar alertas de los peligros de los directivos que perduran demasiado tiempo en sus cargos, pero por otro lado, el directivo que deja su cargo antes de cuatro o cinco años no ha tenido la oportunidad de lograr su rendimiento máximo y puede ser especialmente dañino para el sector público acumular una serie de directivos con mandatos cortos, cada uno de los cuales introduce una nueva dirección, es decir, según (Hambrick y Fukutomi, 1991) citado por (Ysa y Salvador, 2015), las organizaciones deberían establecer horizontes entre cinco y diez años para sus directivos públicos.

La alta rotación del personal directivo o de nivel jerárquico superior, podría ser perjudicial para las instituciones del sector público ya que interrumpe la planificación preestablecida, incrementa los tiempos programados y puede llegar a reducir la eficiencia, por lo que es importante definir de acuerdo al ciclo de vida del cargo directivo un periodo adecuado que permita su desarrollo, así como alcanzar el rendimiento máximo en la institución y la ejecución de la planificación establecida durante dicho periodo.

(Corzo, Coronel, Arlet, Suca, y Velásquez, 2018), en su tesis para obtener el grado de Magíster en Administración Estratégica de Empresas sobre Causas de la Rotación Laboral en las Empresas del Sector Asegurador de Cusco, analizaron la relación de la rotación laboral con la satisfacción laboral y el compromiso organizacional, en el mismo citaron el trabajo de (Tariq, Ramzan, y Riaz, 2013), los cuales efectuaron un estudio con la participación de 100 trabajadores de una empresa que consideró tanto la satisfacción laboral como el compromiso organizacional como asuntos relativamente cercanos al desempeño corporativo y por ello propusieron a este último como variable dependiente. Los resultados que consiguieron empleando el análisis de correlación y regresión indicaron que las variables independientes escogidas (rotación laboral, carga de trabajo, cansancio laboral, salario obtenido, y conflictos familiares) mantenían una relación negativa con la variable dependiente.

Así mismo, en el trabajo de grado se cita a (Ahmad y Rainyee, 2014), quienes manifestaron que existía evidencia importante con respecto a la relación estadística de la rotación laboral tanto con la satisfacción laboral como con el compromiso organizacional, y que estos dos últimos aspectos pueden servir para lograr predecir intenciones de abandono de un puesto de trabajo. Además, expresaron que la satisfacción laboral se encontraba correlacionada 
positivamente con el compromiso organizacional y que tal par de aspectos se encontraban correlacionados negativamente con la rotación laboral.

En su estudio concluyeron que si bien la rotación laboral es una cuestión relativamente cotidiana y los niveles de ésta pueden resultar comprensibles hasta cierto punto, el manejo de cifras elevadas al respecto es una señal de la existencia de problemas al interior de una empresa o sector asociados tanto con la satisfacción laboral como con el compromiso organizacional.

En el estudio revisado, se demuestra que existe relación entre las variables rotación de personal y satisfacción laboral, lo cual a su vez puede influir en el desempeño laboral lo que conllevaría a que la institución se aleje de la consecución de sus metas y dificulte la gestión de actividades y los tiempos establecidos del mismo, es importante tomar en cuenta y monitorear continuamente el comportamiento de estas variables en la organización, a través de la medición del clima y satisfacción laboral así como el nivel de rotación de personal, a fin de tomar medidas preventivas y correctivas de ser el caso que permitan garantizar el desarrollo institucional.

A nivel mundial en lo que se refiere al personal de Nivel Jerárquico Superior se han realizado procesos de reformas de las funciones de directivos, en función a las nuevas características y necesidades del entorno, orientada a una función directiva cada vez más profesionalizada, puesto que se ha tomado conciencia de la importancia de los directivos en la gestión pública y la calidad de los servicios públicos, sin embargo, a pesar de estos procesos de reformas, esta variable está limitada por factores políticos que difícilmente podrían superarse. En lo correspondiente a la variable de clima laboral se han realizado diferentes investigaciones dada su importancia en el rendimiento de los trabajadores y por ende en la consecución de los objetivos de las empresas, así como de las instituciones públicas.

En países desarrollados se han realizado investigaciones relacionadas con las variables de estudio, orientados a mejorar sus legislaciones para la ocupación de cargos directivos del sector público que prioricen la profesionalización del personal, conscientes de la importancia del papel de las autoridades no solo como tomadores de decisiones, si no como líderes promotores de cambios en el servicio público.

En el contexto latinoamericano el interés por realizar investigaciones del clima laboral ha crecido rápidamente en los últimos tiempos, pues se ha incluido cada vez más este concepto en el desarrollo organizacional, así como en los indicadores de gestión de las empresas, se han adoptado formas de mediciones establecidas por distintos autores para evaluar el clima laboral de muchas empresas; una de las causas principales para el estudio de esta variable es que se la relaciona estrechamente con conceptos como calidad, productividad, desempeño, eficiencia, eficacia, por toda la importancia que se le ha dado a esta variable se han desarrollado investigaciones que comprueban a través de herramientas metodológicas su incidencia en los conceptos antes mencionados.

A pesar del crecimiento del interés del estudio de estas variables, y de las investigaciones realizadas en la región, no existen suficientes datos o información enfocada al comportamiento y/o relacionamiento de los constructos en las instituciones del sector público, si bien los conceptos son universales y se aplican tanto al sector público como al privado, existen especificidades dentro del sector gubernamental que requieren de investigación y análisis. 
Sin embargo, del avance, en Latinoamérica aún no se ha reconocido la importancia del estudio de la relación entre la rotación de personal de Nivel Jerárquico Superior y el Clima Laboral como una estrategia para la mejora de la gestión pública.

La situación a nivel nacional es similar, los estudios del clima laboral y su incidencia en el desarrollo organizacional de empresas privadas se ha incrementado en los últimos años, sin embargo, en el sector público, aún no se han evidenciado investigaciones orientadas al estudio de las especificidades de este sector. La alta rotación del personal de nivel jerárquico superior es un problema generalizado en el sector público, por su naturaleza y base legal en Ecuador, de acuerdo con la (Ley Orgánica de Servicio Público, Registro Oficial Suplemento 294 de 06-oct.2010, 2010), artículo 83.- Servidoras y servidores públicos excluidos de la carrera del servicio público.- Exclúyase del sistema de la carrera del servicio público, a: a.3 Las o los secretarios y subsecretarios comprendidos en el nivel jerárquico superior, no existe un plan de carrera para que los funcionarios públicos puedan a través de ascensos acceder a estos cargos directivos, así mismo a pesar de que de conformidad con lo establecido en el (Reglamento General a la Ley Orgánica del Servicio Público, Registro Oficial Suplemento 418 de 01-abr.-2011, 2011), en su artículo 171.- "Descripción y valoración de los puestos del nivel jerárquico superior.- Los puestos del nivel jerárquico superior serán descritos y valorados para garantizar su clasificación adecuada; equidad en la aplicación del sistema integrado del talento humano en las instituciones del Estado; y, servirá para que los ocupantes de los mismos reúnan los requisitos de orden general que sean exigidos para su desempeño, de ser el caso, conforme la norma emitida por el Ministerio de Relaciones Laborales", en la práctica no se cumple, pues el personal directivo se designa más que por experiencia o conocimientos, por asuntos de carácter político, lo cual supone un problema constante que afecta al clima laboral de las entidades públicas que deben enfrentar permanentemente esta situación, por esta razón es importante identificar como influye la rotación del personal de Nivel Jerárquico Superior (NJS) en el clima laboral de las instituciones públicas y definir acciones que permitan minimizar los efectos negativos en la variable clima laboral.

La alta rotación del personal de Nivel Jerárquico Superior supone la ausencia o inestabilidad de una figura de liderazgo que debe ser indispensable y permanente en toda institución, ya sea pública o privada, pues permite mantener un orden, claridad y constancia en las actividades que se realizan para alcanzar eficazmente el objetivo que persigue la institución.

Es importante destacar la relevancia de la estabilidad y permanencia del talento humano considerado de nivel jerárquico superior en las instituciones gubernamentales, puesto que permitirá elevar la efectividad de la gestión pública.

\section{Problemática}

La importancia del estudio de las variables rotación de personal de Nivel Jerárquico Superior y clima laboral se da por cuanto influyen en el comportamiento, actuaciones, reacciones y sentimientos de las personas en su lugar de trabajo; al ser el sector público un sector orientado a la dotación de servicios y satisfacción de necesidades de los ciudadanos es de gran importancia 
garantizar la calidad y eficiencia en la provisión de dichos servicios y la satisfacción de la ciudadanía del país.

Se considera un tema de actualidad con base en lo expuesto anteriormente, puesto que se acoge a lo establecido en el actual (Plan Nacional de Desarrollo, 2017) que contempla dentro de sus objetivos la institucionalización de una administración pública democrática, participativa, incluyente, intercultural y orientada hacia la ciudadanía, basada en un servicio meritocrático profesionalizado que se desempeñe en condiciones dignas, entendiéndose que al referirse a "condiciones dignas" en el mismo se encuentra implícito el concepto de clima laboral y en los términos "servicio meritocrático profesionalizado" el de rotación de personal de nivel jerárquico superior.

Solo durante el año 2018, en el Servicio de Contratación de Obras (SECOB), se han evidenciado cambios constantes del personal cuyos cargos se consideran del nivel jerárquico superior. En la institución a nivel nacional, en promedio se cambian autoridades (sean estos directores, secretarios, subsecretarios, coordinadores, coordinadores zonales, etc.) cada 2,27 meses, las autoridades en cargos de nivel jerárquico superior se han cambiado hasta 5 veces en el mencionado periodo en un mismo Departamento o Dirección; considerando que la misión de la institución es la de contratar, ejecutar y fiscalizar obras de infraestructura pública, por la naturaleza e importancia de los cargos directivos como tomadores de decisiones en la organización, es necesario procurar un nivel adecuado de rotación que no exceda los niveles óptimos que permitan el crecimiento y desarrollo de la institución de acuerdo con los planes, programas y tiempos establecidos para su ejecución.

Adicionalmente un factor de relevancia para la realización de la presente investigación es que en la institución existe antecedente de un estudio de este tipo, que analiza la incidencia de la rotación de personal de nivel jerárquico superior en el clima laboral, por lo cual es importante contrastar los resultados del estudio y comportamiento de estas variables, a fin de plantear y adoptar acciones correctivas y de mejora que permitan garantizar un clima laboral adecuado para los servidores de la institución y por ende el desempeño óptimo de sus funciones, así como de los procesos gobernantes, adjetivos y sustantivos y sentar un precedente para el desarrollo de futuras investigaciones que permitan ampliar la visión y el conocimiento sobre los constructos y su importancia en la mejora de la gestión en el sector público.

\section{Metodología}

Para la investigación se determina que la población del Servicio de Contratación de Obras SECOB es 281. Una vez aplicada la fórmula, se obtiene que el tamaño de la muestra a la cual se aplicará el instrumento de recolección y análisis de información es de 163.

Para la validación del instrumento de medición del clima laboral, se realizó la revisión de investigaciones de varios autores con estudios relacionados con la variable, se escogió el instrumento utilizado en el artículo. El clima organizacional y la satisfacción laboral: un análisis cuantitativo riguroso de su relación de (Manosalvas, Manosalvas, y Quintero, 2015), se solicitó 
el mismo vía correo electrónico, teniendo una respuesta favorable y se procedió a aplicarlo en la muestra obtenida, el instrumento consta de 53 preguntas que miden la percepción del clima laboral de los funcionarios de la institución en 7 dimensiones: comunicación interna (14 ítems), reconocimiento (10 ítems), relaciones interpersonales en el trabajo (8 ítems), toma de decisiones (6 ítems), entorno físico (6 ítems), compromiso (4 ítems) y adaptación al cambio (5 ítems). Se utilizó la escala de respuesta de Likert de 5 puntos: muy de acuerdo (5), de acuerdo (4), no estoy seguro (3), en desacuerdo (2), totalmente en desacuerdo (1).

Para la medición de la rotación del personal de nivel jerárquico superior, en el cuestionario se incluyeron dos preguntas relacionadas con la variable, la primera relacionada al cambio o no de jefe directo en cada área de la institución, a través de una escala dicotómica (si/no) a fin de obtener el porcentaje de rotación; la segunda relacionada con el número de veces que se ha cambiado al personal directivo durante el año 2018, año escogido para el análisis por evidenciar un alto número de cambios de jefes registrados en dicho periodo.

La validez de los instrumentos, para el presente trabajo de investigación, se realizó mediante la técnica de "juicio de expertos". Ellos analizan el instrumento bajo cinco conceptos: claridad, objetividad, consistencia, pertinencia y suficiencia, dentro de cada concepto se realiza una valoración entre muy malo (1) y muy bueno (5), una vez valorado se obtiene la sumatoria parcial y total, posteriormente se detallan los resultados de la validación y la opinión, dentro de las cuales se escoge si es favorable, debe mejorar o no es favorable y finalmente se realizan las observaciones que cada experto considere pertinente con su respectiva firma. Si el instrumento es favorable, puede ser aplicado en la muestra para el levantamiento de la información.

Para determinar la confiabilidad de las escalas que se utilizaron para medir la variable clima organizacional se calculó el índice del alfa de Cronbach. Para que una escala sea considerada confiable debe obtener un valor de alfa mayor o igual a 0.7 (DeVellis, 2003). Los resultados que se muestran en la tabla evidencian que el índice obtenido es de 0,962 permitiendo concluir que la escala es bastante confiable para medir las variables analizadas, como se muestra en la tabla 1.

Tabla 1

Confiabilidad del instrumento
Alfa de Cronbach
No. de elementos

,962 53

\section{Aplicación del instrumento}

El proceso para aplicación del instrumento es el siguiente:

Se obtuvo la nómina de funcionarios de la institución a través de la Dirección de Administración de Talento Humano a fin de conocer la población y obtener la muestra a la cual se aplicará el instrumento. 
Una vez obtenida la muestra y el instrumento validado se solicitó autorización a la Máxima Autoridad de la institución para proceder con la aplicación del instrumento de recolección de información.

Con la debida autorización se procedió a aplicar el instrumento de recolección de información que en la presente investigación es la encuesta, en los diferentes departamentos de la institución junto con el personal de la Dirección de Administración de Talento Humano delegado para el efecto, con la encuesta se pretende recabar información que permita determinar si existe relación entre las variables de estudio: rotación de personal de nivel jerárquico superior y clima laboral.

\section{Resultados}

Una vez recopilada la información a través de las encuestas aplicadas a los funcionarios de la institución se procedió a tabular las respuestas de cada una de las dimensiones medidas dentro de la variable clima laboral.

\section{Tabla 2}

\section{Dimensión comunicación interna}

\begin{tabular}{|c|c|c|c|c|c|c|c|c|c|c|c|}
\hline \multirow[t]{2}{*}{ No. } & \multirow[t]{2}{*}{ Pregunta } & \multicolumn{2}{|c|}{$\begin{array}{l}\text { Muy de } \\
\text { acuerdo }\end{array}$} & \multicolumn{2}{|c|}{$\begin{array}{c}\text { De } \\
\text { acuerdo }\end{array}$} & \multicolumn{2}{|c|}{$\begin{array}{c}\text { No } \\
\text { estoy } \\
\text { seguro }\end{array}$} & \multicolumn{2}{|c|}{$\begin{array}{c}\text { En } \\
\text { desacuerdo }\end{array}$} & \multicolumn{2}{|c|}{$\begin{array}{l}\text { Totalmente } \\
\text { en } \\
\text { desacuerdo }\end{array}$} \\
\hline & & $\mathbf{F}$ & $\%$ & $\mathbf{F}$ & $\%$ & $\mathbf{F}$ & $\%$ & $\mathbf{F}$ & $\%$ & $\mathbf{F}$ & $\%$ \\
\hline 1 & $\begin{array}{l}\text { ¿Se siente Ud. libre para conversar } \\
\text { con su superior acerca de materias } \\
\text { relacionadas con su trabajo? }\end{array}$ & 76 & 47 & 71 & 44 & 10 & 6 & 3 & 2 & 3 & 2 \\
\hline 2 & $\begin{array}{l}\text { ¿Es adecuada la relación con sus } \\
\text { compañeros de trabajo? }\end{array}$ & 72 & 44 & 74 & 45 & 12 & 7 & 5 & 3 & 0 & 0 \\
\hline 3 & $\begin{array}{c}\text { ¿Siente que su relación con su jefe } \\
\text { directo es de confianza y apoyo } \\
\text { recíproco? }\end{array}$ & 77 & 47 & 59 & 36 & 20 & 12 & 3 & 2 & 4 & 2 \\
\hline 4 & $\begin{array}{c}\text { ¿Cree Ud. que en su área de trabajo } \\
\text { se producen errores por falta de } \\
\text { información? }\end{array}$ & 25 & 15 & 77 & 47 & 31 & 19 & 20 & 12 & 10 & 6 \\
\hline 5 & $\begin{array}{c}\text { ¿Su jefe se preocupa de explicar todo } \\
\text { muy bien de modo que no existan } \\
\text { confusiones? }\end{array}$ & 53 & 33 & 81 & 50 & 19 & 12 & 7 & 4 & 3 & 2 \\
\hline 6 & $\begin{array}{c}\text { ¿Considera Ud. que se le entrega la } \\
\text { información suficiente en el } \\
\text { momento, para realizar bien su } \\
\text { trabajo? }\end{array}$ & 29 & 18 & 72 & 44 & 42 & 26 & 14 & 9 & 6 & 4 \\
\hline 7 & $\begin{array}{l}\text { ¿Ante un problema en su trabajo } \\
\text { puede Ud. hablar con su jefe en } \\
\text { forma franca y sincera? }\end{array}$ & 84 & 52 & 49 & 30 & 24 & 15 & 3 & 2 & 3 & 2 \\
\hline 8 & ¿Trabajamos en equipo? & 64 & 39 & 61 & 37 & 31 & 19 & 5 & 3 & 2 & 1 \\
\hline 9 & ¿Avanzamos en la misma dirección? & 63 & 39 & 57 & 35 & 32 & 20 & 9 & 6 & 2 & 1 \\
\hline 10 & $\begin{array}{l}\text { ¿Nuestra información se comparte } \\
\text { con el resto del equipo de trabajo? }\end{array}$ & 62 & 38 & 63 & 39 & 28 & 17 & 8 & 5 & 2 & 1 \\
\hline
\end{tabular}




\begin{tabular}{|c|c|c|c|c|c|c|c|c|c|c|c|}
\hline \multirow[t]{2}{*}{ No. } & \multirow[t]{2}{*}{ Pregunta } & \multicolumn{2}{|c|}{$\begin{array}{l}\text { Muy de } \\
\text { acuerdo }\end{array}$} & \multicolumn{2}{|c|}{$\begin{array}{c}\text { De } \\
\text { acuerdo }\end{array}$} & \multicolumn{2}{|c|}{$\begin{array}{c}\text { No } \\
\text { estoy } \\
\text { seguro }\end{array}$} & \multicolumn{2}{|c|}{$\begin{array}{c}\text { En } \\
\text { desacuerdo }\end{array}$} & \multicolumn{2}{|c|}{$\begin{array}{c}\text { Totalmente } \\
\text { en } \\
\text { desacuerdo }\end{array}$} \\
\hline & & $\mathbf{F}$ & $\%$ & $\mathbf{F}$ & $\%$ & $\mathbf{F}$ & $\%$ & $\mathbf{F}$ & $\%$ & $\mathbf{F}$ & $\%$ \\
\hline 11 & $\begin{array}{c}\text { ¿Nuestros mecanismos de } \\
\text { comunicación son adecuados? }\end{array}$ & 43 & 26 & 76 & 47 & 32 & 20 & 10 & 6 & 2 & 1 \\
\hline 12 & ¿Realizamos reuniones efectivas? & 37 & 23 & 73 & 45 & 36 & 22 & 13 & 8 & 4 & 2 \\
\hline 13 & $\begin{array}{c}\text { ¿Comunicamos entre nosotros } \\
\text { cuando y donde podemos ser } \\
\text { localizados? }\end{array}$ & 45 & 28 & 83 & 51 & 29 & 18 & 6 & 4 & 0 & 0 \\
\hline \multirow[t]{4}{*}{14} & $\begin{array}{c}\text { ¿Respetamos nuestro tiempo } \\
\text { mutuamente? }\end{array}$ & 49 & 30 & 73 & 45 & 30 & 18 & 7 & 4 & 4 & 2 \\
\hline & Frecuencia & 779 & & 969 & & 376 & & 113 & & 45 & \\
\hline & Promedio & 56 & & 69 & & 27 & & 8 & & 3 & \\
\hline & Porcentaje & 34 & & 42 & & 16 & & 5 & & 2 & \\
\hline
\end{tabular}

De acuerdo con la tabla 2 que corresponde a la dimensión comunicación interna, al obtener el promedio de las frecuencias de cada una de las preguntas, se observa que, del total de encuestados (163), el 34\% se encuentra muy de acuerdo con los elementos considerados dentro de la comunicación interna de la institución, mientras que el $42 \%$, está de acuerdo, el $16 \%$ no está seguro, el $5 \%$ está en desacuerdo y el $2 \%$ está totalmente en desacuerdo.

Con los resultados obtenidos, se tiene que en general la percepción de los funcionarios de la institución respecto de la comunicación interna es que existen condiciones adecuadas que promueven una efectiva comunicación en la organización, como el trabajo en equipo, adecuada relación entre compañeros de trabajo y la confianza en el jefe directo, sin embargo de ello, de acuerdo con la información obtenida se muestra que existe contradicción en las respuestas correspondientes a la entrega de información suficiente para el desarrollo del trabajo, por lo que se evidencia que no existe claridad de parte de los funcionarios de la institución en cuanto a este aspecto.

\section{Tabla 3}

\section{Dimensión reconocimiento}

\begin{tabular}{|c|c|c|c|c|c|c|c|c|c|c|c|}
\hline \multirow[t]{2}{*}{ No. } & \multirow[t]{2}{*}{ Pregunta } & \multicolumn{2}{|c|}{$\begin{array}{l}\text { Muy de } \\
\text { acuerdo }\end{array}$} & \multicolumn{2}{|c|}{ De acuerdo } & \multicolumn{2}{|c|}{$\begin{array}{l}\text { No estoy } \\
\text { seguro }\end{array}$} & \multicolumn{2}{|c|}{$\begin{array}{c}\text { En } \\
\text { desacuerdo }\end{array}$} & \multicolumn{2}{|c|}{$\begin{array}{c}\text { Totalmente } \\
\text { en } \\
\text { desacuerdo }\end{array}$} \\
\hline & & $\mathbf{F}$ & $\%$ & $\mathbf{F}$ & $\%$ & $\mathbf{F}$ & $\%$ & $\mathbf{F}$ & $\%$ & $\mathbf{F}$ & $\%$ \\
\hline 1 & $\begin{array}{l}\text { ¿Se estimula la capacitación } \\
\text { de los funcionarios? }\end{array}$ & 36 & 22 & 58 & 36 & 39 & 24 & 24 & 15 & 6 & 4 \\
\hline 2 & $\begin{array}{l}\text { ¿Se trabaja regularmente con } \\
\text { un plan para la capacitación de } \\
\text { los funcionarios? }\end{array}$ & 37 & 23 & 59 & 36 & 37 & 23 & 24 & 15 & 6 & 4 \\
\hline 3 & $\begin{array}{c}\text { ¿Se ofrecen posibilidades } \\
\text { para el desarrollo personal de } \\
\text { los funcionarios? }\end{array}$ & 21 & 13 & 45 & 28 & 53 & 33 & 32 & 20 & 12 & 7 \\
\hline 4 & $\begin{array}{l}\text { ¿Existe equidad en el acceso } \\
\text { a la capacitación? }\end{array}$ & 29 & 18 & 54 & 33 & 48 & 29 & 25 & 15 & 7 & 4 \\
\hline
\end{tabular}




\begin{tabular}{|c|c|c|c|c|c|c|c|c|c|c|c|}
\hline \multirow[t]{2}{*}{ No. } & \multirow[t]{2}{*}{ Pregunta } & \multicolumn{2}{|c|}{$\begin{array}{l}\text { Muy de } \\
\text { acuerdo }\end{array}$} & \multicolumn{2}{|c|}{ De acuerdo } & \multicolumn{2}{|c|}{$\begin{array}{l}\text { No estoy } \\
\text { seguro }\end{array}$} & \multicolumn{2}{|c|}{$\begin{array}{c}\text { En } \\
\text { desacuerdo }\end{array}$} & \multicolumn{2}{|c|}{$\begin{array}{c}\text { Totalmente } \\
\text { en } \\
\text { desacuerdo }\end{array}$} \\
\hline & & $\mathbf{F}$ & $\%$ & $\mathbf{F}$ & $\%$ & $\mathbf{F}$ & $\%$ & $\mathbf{F}$ & $\%$ & $\mathbf{F}$ & $\%$ \\
\hline 5 & $\begin{array}{c}\text { ¿Los funcionarios de su } \\
\text { Departamento/ Servicio/ } \\
\text { Unidad son frecuentemente } \\
\text { elogiados, más que criticados } \\
\text { y corregidos? }\end{array}$ & 16 & 10 & 59 & 36 & 58 & 36 & 21 & 13 & 9 & 6 \\
\hline 6 & $\begin{array}{l}\text { ¿Hemos sido partícipes de } \\
\text { los buenos resultados? }\end{array}$ & 37 & 23 & 71 & 44 & 36 & 22 & 11 & 7 & 8 & 5 \\
\hline 7 & $\begin{array}{l}\text { ¿La actuación de calidad es } \\
\text { gratificada? }\end{array}$ & 21 & 13 & 49 & 30 & 56 & 34 & 24 & 15 & 13 & 8 \\
\hline 8 & $\begin{array}{l}\text { ¿La jefatura se fija más en } \\
\text { las habilidades de los } \\
\text { funcionarios que en sus puntos } \\
\text { débiles? }\end{array}$ & 24 & 15 & 70 & 43 & 52 & 32 & 9 & 6 & 8 & 5 \\
\hline 9 & $\begin{array}{l}\text { ¿Hay grupos de funcionarios } \\
\text { que casi nunca reciben } \\
\text { atención ni reconocimiento? }\end{array}$ & 20 & 12 & 59 & 36 & 51 & 31 & 18 & 11 & 15 & 9 \\
\hline 10 & $\begin{array}{l}\text { ¿El funcionario que ha sido } \\
\text { llamado por el jefe, espera más } \\
\text { bien elogios que críticas? }\end{array}$ & 12 & 7 & 54 & 33 & 67 & 41 & 21 & 13 & 9 & 6 \\
\hline & Frecuencia & 253 & & 578 & & 497 & & 209 & & 93 & \\
\hline & Promedio & 25 & & 58 & & 50 & & 21 & & 9 & \\
\hline & Porcentaje & 16 & & 35 & & 30 & & 13 & & 6 & \\
\hline
\end{tabular}

En la dimensión reconocimiento de acuerdo con el promedio obtenido de las respuestas, el $16 \%$ estuvo muy de acuerdo, $35 \%$ de acuerdo, $30 \%$ no está seguro, $13 \%$ no está de acuerdo y $6 \%$ está totalmente en desacuerdo.

Con respecto a la dimensión reconocimiento se consideran elementos como capacitación, desarrollo personal, participación de resultados, gratificaciones y atención a los funcionarios, con los resultados obtenidos se puede observar que a pesar de que la mayor parte de encuestados está de acuerdo con lo consultado, existe igualmente un alto porcentaje de funcionarios que responden no estar seguros, lo que podría interpretarse como desconocimiento en temas de reconocimiento o no existe claridad en las políticas de gratificación y recompensa con las que cuenta la institución.

\section{Tabla 4}

Dimensión relaciones interpersonales en el trabajo

\begin{tabular}{|c|c|c|c|c|c|c|c|c|c|c|c|}
\hline \multirow[t]{2}{*}{ No. } & \multirow[t]{2}{*}{ Pregunta } & \multicolumn{2}{|c|}{$\begin{array}{l}\text { Muy de } \\
\text { acuerdo }\end{array}$} & \multicolumn{2}{|c|}{ De acuerdo } & \multicolumn{2}{|c|}{$\begin{array}{l}\text { No estoy } \\
\text { seguro }\end{array}$} & \multicolumn{2}{|c|}{$\begin{array}{c}\text { En } \\
\text { desacuerdo }\end{array}$} & \multicolumn{2}{|c|}{$\begin{array}{l}\text { Totalmente } \\
\text { en } \\
\text { desacuerdo }\end{array}$} \\
\hline & & $\mathbf{F}$ & $\%$ & $\mathbf{F}$ & $\%$ & $\mathbf{F}$ & $\%$ & $\mathbf{F}$ & $\%$ & $\mathbf{F}$ & $\%$ \\
\hline 1 & $\begin{array}{c}\text { ¿Lo pasamos bien trabajando } \\
\text { juntos? }\end{array}$ & 62 & 38 & 76 & 47 & 19 & 12 & 6 & 4 & 0 & 0 \\
\hline 2 & $\begin{array}{l}\text { ¿Tenemos ganas de ir a } \\
\text { trabajar cada día? }\end{array}$ & 67 & 41 & 64 & 39 & 22 & 13 & 6 & 4 & 4 & 2 \\
\hline
\end{tabular}




\begin{tabular}{|c|c|c|c|c|c|c|c|c|c|c|c|}
\hline \multirow[t]{2}{*}{ No. } & \multirow[t]{2}{*}{ Pregunta } & \multicolumn{2}{|c|}{$\begin{array}{l}\text { Muy de } \\
\text { acuerdo }\end{array}$} & \multicolumn{2}{|c|}{ De acuerdo } & \multicolumn{2}{|c|}{$\begin{array}{l}\text { No estoy } \\
\text { seguro }\end{array}$} & \multicolumn{2}{|c|}{$\begin{array}{c}\text { En } \\
\text { desacuerdo }\end{array}$} & \multicolumn{2}{|c|}{$\begin{array}{c}\text { Totalmente } \\
\text { en } \\
\text { desacuerdo }\end{array}$} \\
\hline & & $\mathbf{F}$ & $\%$ & $\mathbf{F}$ & $\%$ & $\mathbf{F}$ & $\%$ & $\mathbf{F}$ & $\%$ & $\mathbf{F}$ & $\%$ \\
\hline 3 & $\begin{array}{l}\text { ¿Nos sentimos seguros en } \\
\text { nuestro trabajo? }\end{array}$ & 35 & 21 & 45 & 28 & 30 & 18 & 23 & 14 & 30 & 18 \\
\hline 4 & $\begin{array}{c}\text { ¿Nos ayudamos y animamos } \\
\text { unos a otros? }\end{array}$ & 56 & 34 & 69 & 42 & 29 & 18 & 6 & 4 & 3 & 2 \\
\hline 5 & $\begin{array}{l}\text { ¿Nuestra comunicación es } \\
\text { abierta y transparente? }\end{array}$ & 49 & 30 & 69 & 42 & 33 & 20 & 6 & 4 & 6 & 4 \\
\hline 6 & $\begin{array}{c}\text { ¿Confiamos los unos en los } \\
\text { otros? }\end{array}$ & 36 & 22 & 64 & 39 & 40 & 25 & 14 & 9 & 9 & 6 \\
\hline 7 & $\begin{array}{l}\text { ¿Hablamos los unos CON los } \\
\text { otros, pero no los unos DE los } \\
\text { otros? }\end{array}$ & 35 & 21 & 63 & 39 & 42 & 26 & 14 & 9 & 9 & 6 \\
\hline 8 & $\begin{array}{l}\text { ¿Respetamos las habilidades, } \\
\text { los deseos y la personalidad de } \\
\text { los demás? }\end{array}$ & 58 & 36 & 77 & 47 & 21 & 13 & 3 & 2 & 4 & 2 \\
\hline & Frecuencia & 398 & & 495 & & 235 & & 73 & & 64 & \\
\hline & Promedio & 50 & & 62 & & 29 & & 9 & & 8 & \\
\hline & Porcentaje & 31 & & 38 & & 18 & & 6 & & 5 & \\
\hline
\end{tabular}

Dentro de la dimensión relaciones interpersonales en el trabajo se consideran aspectos como seguridad, motivación, ayuda y colaboración mutua, confianza, comunicación transparente y respeto, el 29\% de los encuestados respondió estar muy de acuerdo, el $41 \%$ está de acuerdo, el $19 \%$ no está seguro, el $6 \%$ está en desacuerdo y el 5\% está totalmente en desacuerdo.

Con estos resultados en términos generales se evidencia que existe un buen número de funcionarios de la institución que se encuentran satisfechos con las relaciones interpersonales en el trabajo, es decir que perciben un ambiente de respeto, confianza y apoyo mutuo entre compañeros.

\section{Tabla 5}

\section{Dimensión toma de decisiones}

\begin{tabular}{|c|c|c|c|c|c|c|c|c|c|c|c|}
\hline \multirow[t]{2}{*}{ No. } & \multirow[t]{2}{*}{ Pregunta } & \multicolumn{2}{|c|}{$\begin{array}{l}\text { Muy de } \\
\text { acuerdo }\end{array}$} & \multicolumn{2}{|c|}{ De acuerdo } & \multicolumn{2}{|c|}{$\begin{array}{l}\text { No estoy } \\
\text { seguro }\end{array}$} & \multicolumn{2}{|c|}{$\begin{array}{c}\text { En } \\
\text { desacuerdo }\end{array}$} & \multicolumn{2}{|c|}{$\begin{array}{c}\text { Totalmente } \\
\text { en } \\
\text { desacuerdo }\end{array}$} \\
\hline & & $\mathbf{F}$ & $\%$ & $\mathbf{F}$ & $\%$ & $\mathbf{F}$ & $\%$ & $\mathbf{F}$ & $\%$ & $\mathbf{F}$ & $\%$ \\
\hline 1 & $\begin{array}{l}\text { ¿Las decisiones de su } \\
\text { Departamento/ Servicio/ } \\
\text { Unidad se toman en su } \\
\text { mismo nivel? }\end{array}$ & 26 & 16 & 76 & 47 & 41 & 25 & 15 & 9 & 5 & 3 \\
\hline 2 & $\begin{array}{c}\text { ¿Las decisiones se toman } \\
\text { oportunamente? }\end{array}$ & 43 & 26 & 63 & 39 & 38 & 23 & 15 & 9 & 4 & 2 \\
\hline 3 & $\begin{array}{l}\text { Piensa Ud. que las decisiones } \\
\text { en su unidad se hacen } \\
\text { pensando en los trabajadores. }\end{array}$ & 29 & 18 & 67 & 41 & 32 & 20 & 20 & 12 & 15 & 9 \\
\hline
\end{tabular}




\begin{tabular}{|c|c|c|c|c|c|c|c|c|c|c|c|}
\hline \multirow[t]{2}{*}{ No. } & \multirow[t]{2}{*}{ Pregunta } & \multicolumn{2}{|c|}{$\begin{array}{l}\text { Muy de } \\
\text { acuerdo }\end{array}$} & \multicolumn{2}{|c|}{ De acuerdo } & \multicolumn{2}{|c|}{$\begin{array}{c}\text { No estoy } \\
\text { seguro }\end{array}$} & \multicolumn{2}{|c|}{$\begin{array}{c}\text { En } \\
\text { desacuerdo }\end{array}$} & \multicolumn{2}{|c|}{$\begin{array}{c}\text { Totalmente } \\
\text { en } \\
\text { desacuerdo }\end{array}$} \\
\hline & & $\mathbf{F}$ & $\%$ & $\mathbf{F}$ & $\%$ & $\mathbf{F}$ & $\%$ & $\mathbf{F}$ & $\%$ & $\mathbf{F}$ & $\%$ \\
\hline 4 & $\begin{array}{l}\text { Ud. Puede emplear su propia } \\
\text { iniciativa para hacer las } \\
\text { cosas. }\end{array}$ & 43 & 26 & 74 & 45 & 26 & 16 & 15 & 9 & 5 & 3 \\
\hline 5 & $\begin{array}{c}\text { Siente Ud. que en esta } \\
\text { organización se anima los } \\
\text { empleados a que tomen sus } \\
\text { propias decisiones }\end{array}$ & 22 & 13 & 60 & 37 & 43 & 26 & 22 & 13 & 16 & 10 \\
\hline \multirow[t]{4}{*}{6} & $\begin{array}{l}\text { ¿Las decisiones se basan en } \\
\text { información confiable? }\end{array}$ & 40 & 25 & 67 & 41 & 37 & 23 & 13 & 8 & 6 & 4 \\
\hline & Frecuencia & 203 & & 407 & & 217 & & 100 & & 51 & \\
\hline & Promedio & 34 & & 68 & & 36 & & 17 & & 9 & \\
\hline & Porcentaje & 21 & & 42 & & 22 & & 10 & & 5 & \\
\hline
\end{tabular}

En esta dimensión los encuestados respondieron estar muy de acuerdo $21 \%$, de acuerdo $42 \%$, no está seguro $22 \%$, en desacuerdo $10 \%$, totalmente en desacuerdo $5 \%$, lo que evidencia que los resultados se concentran en su mayoría (63\%) en las respuestas de acuerdo y muy de acuerdo.

La dimensión toma de decisiones incluye aspectos referentes al incentivo, oportunidad, igualdad e iniciativa en la toma de decisiones de la empresa y la inclusión de sus funcionarios en la misma, de acuerdo con los resultados obtenidos se observa que la percepción de los empleados en cuanto a esta dimensión es buena, lo que significa que las condiciones que presta la institución en cuanto a toma de decisiones considera la opinión de los funcionarios, se propicia su iniciativa y se basa en información confiable.

\section{Tabla 6}

Dimensión entorno físico

\begin{tabular}{|c|c|c|c|c|c|c|c|c|c|c|c|}
\hline \multirow[t]{2}{*}{ No. } & \multirow[t]{2}{*}{ Pregunta } & \multicolumn{2}{|c|}{$\begin{array}{l}\text { Muy de } \\
\text { acuerdo }\end{array}$} & \multicolumn{2}{|c|}{$\begin{array}{c}\text { De } \\
\text { acuerdo }\end{array}$} & \multicolumn{2}{|c|}{$\begin{array}{l}\text { No estoy } \\
\text { seguro }\end{array}$} & \multicolumn{2}{|c|}{$\begin{array}{c}\text { En } \\
\text { desacuerdo }\end{array}$} & \multicolumn{2}{|c|}{$\begin{array}{c}\text { Totalmente } \\
\text { en } \\
\text { desacuerdo }\end{array}$} \\
\hline & & $\mathbf{F}$ & $\%$ & $\mathbf{F}$ & $\%$ & $\mathbf{F}$ & $\%$ & $\mathbf{F}$ & $\%$ & $\mathbf{F}$ & $\%$ \\
\hline 1 & $\begin{array}{c}\text { ¿El entorno físico mejora el } \\
\text { bienestar y la eficiencia de su } \\
\text { Departamento/ } \\
\text { Servicio/Unidad? }\end{array}$ & 52 & 32 & 71 & 44 & 26 & 16 & 10 & 6 & 4 & 2 \\
\hline 2 & $\begin{array}{l}\text { La decoración y el diseño: } \\
\text { ¿Son acordes a la función de su } \\
\text { Departamento/ } \\
\text { Servicio/Unidad? }\end{array}$ & 46 & 28 & 67 & 41 & 32 & 20 & 12 & 7 & 6 & 4 \\
\hline 3 & ¿El espacio físico es suficiente? & 56 & 34 & 74 & 45 & 19 & 12 & 8 & 5 & 6 & 4 \\
\hline 4 & $\begin{array}{l}\text { ¿Tiene una ventilación e } \\
\text { iluminación adecuada? }\end{array}$ & 57 & 35 & 71 & 44 & 21 & 13 & 11 & 7 & 3 & 2 \\
\hline
\end{tabular}




\begin{tabular}{|c|c|c|c|c|c|c|c|c|c|c|c|}
\hline \multirow[t]{2}{*}{ No. } & \multirow[t]{2}{*}{ Pregunta } & \multicolumn{2}{|c|}{$\begin{array}{l}\text { Muy de } \\
\text { acuerdo }\end{array}$} & \multicolumn{2}{|c|}{$\begin{array}{c}\text { De } \\
\text { acuerdo }\end{array}$} & \multicolumn{2}{|c|}{$\begin{array}{c}\text { No estoy } \\
\text { seguro }\end{array}$} & \multicolumn{2}{|c|}{$\begin{array}{c}\text { En } \\
\text { desacuerdo }\end{array}$} & \multicolumn{2}{|c|}{$\begin{array}{l}\text { Totalmente } \\
\text { en } \\
\text { desacuerdo }\end{array}$} \\
\hline & & $\mathbf{F}$ & $\%$ & $\mathbf{F}$ & $\%$ & $\mathbf{F}$ & $\%$ & $\mathbf{F}$ & $\%$ & $\mathbf{F}$ & $\%$ \\
\hline 5 & $\begin{array}{l}\text { ¿El nivel de ruido interfiere } \\
\text { significativamente con el } \\
\text { desarrollo de sus funciones? }\end{array}$ & 18 & 11 & 55 & 34 & 29 & 18 & 37 & 23 & 24 & 15 \\
\hline 6 & $\begin{array}{l}\text { ¿Cuenta su Departamento/ } \\
\text { Servicio/ Unidad con los } \\
\text { equipos e insumos necesarios } \\
\text { para el desarrollo de sus } \\
\text { funciones? }\end{array}$ & 50 & 31 & 73 & 28 & 18 & 7 & 16 & 6 & 6 & 2 \\
\hline & Frecuencia & 279 & & 411 & & 145 & & 94 & & 49 & \\
\hline & Promedio & 47 & & 69 & & 24 & & 16 & & 8 & \\
\hline & Porcentaje & 29 & & 42 & & 15 & & 10 & & 5 & \\
\hline
\end{tabular}

Como se muestra en la tabla anterior correspondiente al entorno físico el 29\% respondió estar muy de acuerdo, $42 \%$ de acuerdo, $15 \%$ no está seguro, $10 \%$ en desacuerdo y $5 \%$ totalmente en desacuerdo.

La dimensión se refiere principalmente a los aspectos físicos como decoración y diseño, del entorno como ruido, iluminación y ventilación y equipos tecnológicos e insumos para el desempeño del trabajo diario, con los resultados obtenidos se muestra que la percepción de los funcionarios respecto a esta dimensión es que existen las condiciones físicas adecuadas para el desarrollo del trabajo, es decir que la institución cuenta con la infraestructura y equipamiento necesarios con los que los funcionarios se sienten cómodos.

\section{Tabla 7}

\section{Dimensión compromiso}

\begin{tabular}{|c|c|c|c|c|c|c|c|c|c|c|c|}
\hline \multirow[t]{2}{*}{ No. } & \multirow[t]{2}{*}{ Pregunta } & \multicolumn{2}{|c|}{$\begin{array}{l}\text { Muy de } \\
\text { acuerdo }\end{array}$} & \multicolumn{2}{|c|}{$\begin{array}{c}\text { De } \\
\text { acuerdo }\end{array}$} & \multicolumn{2}{|c|}{$\begin{array}{l}\text { No estoy } \\
\text { seguro }\end{array}$} & \multicolumn{2}{|c|}{$\begin{array}{c}\text { En } \\
\text { desacuerdo }\end{array}$} & \multicolumn{2}{|c|}{$\begin{array}{c}\text { Totalmente } \\
\text { en } \\
\text { desacuerdo }\end{array}$} \\
\hline & & $\mathbf{F}$ & $\%$ & $\mathbf{F}$ & $\%$ & $\mathbf{F}$ & $\%$ & $\mathbf{F}$ & $\%$ & $\mathbf{F}$ & $\%$ \\
\hline 1 & ¿Cumplimos nuestros acuerdos? & 56 & 34 & 90 & 55 & 11 & 7 & 4 & 2 & 2 & 1 \\
\hline 2 & $\begin{array}{c}\text { ¿Estamos dispuestos a hacer un } \\
\text { esfuerzo extra si la situación lo } \\
\text { exige? }\end{array}$ & 83 & 51 & 72 & 44 & 6 & 4 & 1 & 1 & 1 & 1 \\
\hline 3 & $\begin{array}{c}\text { ¿Se siente Ud. con la camiseta } \\
\text { puesta? }\end{array}$ & 84 & 52 & 54 & 33 & 18 & 11 & 4 & 2 & 3 & 2 \\
\hline 4 & $\begin{array}{l}\text { ¿Nos sentimos responsables de los } \\
\text { éxitos y fracasos de nuestro } \\
\text { Departamento/Servicio/ Unidad? }\end{array}$ & 69 & 42 & 70 & 43 & 19 & 12 & 4 & 2 & 1 & 1 \\
\hline & Frecuencia & 292 & & 286 & & 54 & & 13 & & 7 & \\
\hline & Promedio & 73 & & 72 & & 14 & & 3 & & 2 & \\
\hline & Porcentaje & 45 & & 44 & & 8 & & 2 & & 1 & \\
\hline
\end{tabular}

En la tabla 7 se muestran las respuestas obtenidas de los funcionarios respecto de la dimensión compromiso, en la cual se observa que el $45 \%$ respondió estar muy de acuerdo, el 
$44 \%$ de acuerdo, el $8 \%$ no está seguro, el $2 \%$ en desacuerdo y el 1\% totalmente en desacuerdo; al agrupar las respuestas correspondientes a muy de acuerdo y de acuerdo, se obtiene un $89 \%$ del total de encuestados, es decir, 145 funcionarios.

La dimensión compromiso mide aspectos como el cumplimiento de acuerdos, esfuerzo extra, sentirse con la camiseta puesta y sentir como suyos los éxitos o fracasos de la institución, en ésta se evidencia que casi la totalidad de funcionarios se sienten comprometidos con la organización y con la consecución de sus objetivos, lo cual es muy positivo para la institución.

\section{Tabla 8}

Dimensión adaptación al cambio

\begin{tabular}{|c|c|c|c|c|c|c|c|c|c|c|c|}
\hline \multirow[t]{2}{*}{ No. } & \multirow[t]{2}{*}{ Pregunta } & \multicolumn{2}{|c|}{$\begin{array}{l}\text { Muy de } \\
\text { acuerdo }\end{array}$} & \multicolumn{2}{|c|}{$\begin{array}{c}\text { De } \\
\text { acuerdo }\end{array}$} & \multicolumn{2}{|c|}{$\begin{array}{l}\text { No estoy } \\
\text { seguro }\end{array}$} & \multicolumn{2}{|c|}{$\begin{array}{c}\text { En } \\
\text { desacuerdo }\end{array}$} & \multicolumn{2}{|c|}{$\begin{array}{c}\text { Totalmente } \\
\text { en } \\
\text { desacuerdo }\end{array}$} \\
\hline & & $\mathbf{F}$ & $\%$ & $\mathbf{F}$ & $\%$ & $\mathbf{F}$ & $\%$ & $\mathbf{F}$ & $\%$ & $\mathbf{F}$ & $\%$ \\
\hline 1 & $\begin{array}{c}\text { ¿Sabemos cuáles son los } \\
\text { progresos de nuestro } \\
\text { Departamento/ Servicio/ } \\
\text { Unidad? }\end{array}$ & 48 & 29 & 90 & 55 & 20 & 12 & 3 & 2 & 2 & 1 \\
\hline 2 & $\begin{array}{c}\text { ¿Sentimos la necesidad de que } \\
\text { existan cambios? }\end{array}$ & 38 & 23 & 78 & 48 & 26 & 16 & 15 & 9 & 6 & 4 \\
\hline 3 & $\begin{array}{c}\text { ¿Somos lo suficientemente } \\
\text { flexibles para aceptar los } \\
\text { cambios? }\end{array}$ & 59 & 36 & 83 & 51 & 14 & 9 & 6 & 4 & 1 & 1 \\
\hline 4 & $\begin{array}{c}\text { ¿Estamos dispuestos a } \\
\text { adaptarnos a los cambios? }\end{array}$ & 68 & 42 & 78 & 48 & 13 & 8 & 4 & 2 & 0 & 0 \\
\hline 5 & $\begin{array}{c}\text { ¿Hemos empezado a usar nuevas } \\
\text { tecnologías o herramientas de } \\
\text { gestión? }\end{array}$ & 42 & 26 & 60 & 37 & 37 & 23 & 16 & 10 & 8 & 5 \\
\hline & Frecuencia & 255 & & 389 & & 110 & & 44 & & 17 & \\
\hline & Promedio & 51 & & 78 & & 22 & & 9 & & 3 & \\
\hline & Porcentaje & 31 & & 48 & & 13 & & 5 & & 2 & \\
\hline
\end{tabular}

Al aplicar la encuesta en cuanto a la dimensión adaptación al cambio, el 31\% respondió estar muy de acuerdo, el $48 \%$ de acuerdo, el 13\% no está seguro, el 5\% está en desacuerdo y el $2 \%$ totalmente en desacuerdo, agrupando las respuestas muy de acuerdo y de acuerdo se obtiene un $79 \%$ del total de encuestados.

La adaptación al cambio mide aspectos como el conocimiento de los progresos de la institución, la necesidad de que existan cambios, flexibilidad para aceptar cambios y uso de nuevas tecnologías o herramientas de gestión; con las respuestas obtenidas se evidencia que la percepción de los funcionarios en cuanto a esta dimensión es buena, mostrando su satisfacción con la situación en la institución, sin embargo, se observa una contradicción en la pregunta correspondiente a necesidad de que existan cambios en la cual un gran porcentaje de funcionarios responde estar muy de acuerdo y de acuerdo, lo cual no necesariamente refleja 
alguna inconformidad, si no que podría entenderse como cambios relacionados a innovación de procesos y tecnologías.

La hipótesis planteada en la presente investigación es la siguiente:

La rotación de personal de nivel jerárquico superior incide en el clima laboral del Servicio de Contratación de Obras SECOB.

Para la comprobación de la hipótesis, a fin de correlacionar las variables estudiadas, se obtuvo el promedio del clima laboral (Hayes, 2017) incluyendo las siete dimensiones, una vez obtenido el promedio se realizó la comparación de medias con la Prueba T para muestras independientes, al aplicar esta prueba se obtuvo el siguiente resultado:

\section{Tabla 9}

Promedio del clima laboral según rotación de personal de nivel jerárquico superior

\begin{tabular}{cccccc}
\hline & RNJS & N & Media & Desviación estándar & $\begin{array}{c}\text { Media de error } \\
\text { estándar }\end{array}$ \\
\hline PROMEDIO & $\mathrm{Si}$ & 138 & 3,7830 &, 57502 &, 04895 \\
CLIMALABORAL & $\mathrm{No}$ & 25 & 4,0966 &, 48428 &, 09686 \\
\hline
\end{tabular}

\section{Tabla 10}

Prueba de muestras independientes promedio clima laboral

\begin{tabular}{|c|c|c|c|c|c|c|}
\hline & & \multicolumn{2}{|c|}{$\begin{array}{l}\text { Prueba de Levene de } \\
\text { calidad de varianzas }\end{array}$} & \multicolumn{3}{|c|}{$\begin{array}{c}\text { Prueba t para la igualdad de } \\
\text { medias }\end{array}$} \\
\hline & & $\mathbf{F}$ & Sig. & $\mathbf{t}$ & gl & $\begin{array}{c}\text { Sig. } \\
\text { (bilateral) }\end{array}$ \\
\hline Promedio & \multirow{2}{*}{$\begin{array}{c}\text { Se asumen varianzas iguales } \\
\text { No se asumen varianzas iguales }\end{array}$} & 1,465 & ,228 & $-2,565$ & 161 & ,011 \\
\hline $\begin{array}{l}\text { Clima } \\
\text { Laboral }\end{array}$ & & & & $-2,890$ & 37,398 & ,006 \\
\hline
\end{tabular}

Con un valor de p de 0,228 superior a 0,05, no se rechaza la hipótesis nula, de igualdad de varianzas por lo tanto se analiza la prueba $\mathrm{T}$ Student relacionada a varianzas iguales, al analizar la prueba $\mathrm{T}$ se observa que el p valor 0,011 es menor que 0,05 lo que lleva a rechazar la hipótesis de igualdad de medias entre los grupos que tuvieron rotación y los que no la tuvieron. Al analizar el promedio obtenido del clima organizacional en cada uno de los grupos se observa que el grupo que presenta un mejor clima laboral es el que no tuvo una rotación del jerárquico superior $(4,0966)$ en comparación con los que sí tuvieron rotación de jerárquico superior $(3,7830)$, esto permite aceptar la hipótesis de investigación que establece que: La rotación de personal de nivel jerárquico superior incide en el clima laboral del Servicio de Contratación de Obras SECOB. 
Adicionalmente, con el fin de comprobar si existe relación entre cada una de las dimensiones del clima laboral y la rotación del personal de nivel jerárquico superior se obtuvo el promedio de cada dimensión, una vez obtenido el promedio se realizó la comprobación de medias con la Prueba T para muestras independientes, obteniendo los siguientes resultados con cada una de las dimensiones.

\section{Conclusiones}

Con la presente investigación se determinó que existe relación entre las variables estudiadas, es decir la rotación del personal de nivel jerárquico superior incide en el clima laboral del Servicio de Contratación de Obras SECOB, pues de acuerdo con los resultados obtenidos, se evidenció que existe un mejor clima laboral en el grupo que no ha experimentado cambios de directivos, a diferencia de los que sí lo han experimentado.

A pesar de que durante el desarrollo de toda la investigación se han estudiado teorías que permiten sustentar la relación entre la rotación del personal y el clima laboral, no existen suficientes estudios o investigaciones que analicen a fondo la rotación específicamente del personal de nivel jerárquico superior en el sector público y su incidencia en el clima laboral, lo cual no ha permitido conocer de mejor manera las especificidades de estas variables aplicadas al sector público, en su gran mayoría se han realizado investigaciones en el sector privado, sin embargo, los resultados en el sector privado no suelen ser los mismos en el sector público por sus características y especificidades.

Como resultado de la presente investigación se pudo determinar el estado de las variables de estudio en la institución, es así que, se observó que existe una alta rotación del personal de nivel jerárquico superior en el Servicio de Contratación de Obras SECOB, específicamente durante el año 2018, en el cual se encontró que del total de encuestados (163), el 85\% que corresponde a 145 funcionarios, experimentó cambios de directivos al menos una vez en ese periodo.

Al analizar los resultados obtenidos con la investigación se observó que, existe diferencia de medias entre los grupos que experimentaron cambios de directivos y los que no, al tener significancia estadística son aplicables a la población; así mismo, al analizar cada una de las siete dimensiones medidas con la encuesta, se observa que no existe incidencia de la rotación de jerárquico superior en tres de ellas (comunicación interna, entorno físico y adaptación al cambio), mientras que en las cuatro dimensiones restantes (reconocimiento, relaciones interpersonales en el trabajo, toma de decisiones, compromiso) se evidencia que sí existe incidencia pues la alta rotación de jefes ocasiona un cambio constante en el grupo de trabajo, los funcionarios no se sienten parte dentro del grupo, sienten inestabilidad e incertidumbre lo cual influye en el clima laboral.

A pesar de la importancia del clima laboral en el desempeño de toda organización pública o privada para la consecución de sus objetivos, actualmente su estudio y análisis no se considera una prioridad en el sector público, si bien se realizan encuestas que miden el clima laboral, no se 
adoptan medidas y políticas, que permitan corregir y mejorar las falencias detectadas en los resultados de las encuestas aplicadas.

La rotación del personal de nivel jerárquico superior constituye un factor importante en la medición del clima laboral, sin embargo, en el sector público, por su naturaleza y de acuerdo con la normativa legal vigente, existe un alto porcentaje de rotación de directivos, sin que se le haya atribuido la relevancia real que tiene, por lo que no se han realizado acciones para minimizar su impacto en la institución.

\section{Referencias Bibliográficas}

Ahmad, A., \& Rainyee, R. (2014). Which is the best predictor of employee turnover intentions: Job satisfaction or organizational commitment? A literature review. International Journal of Information, Business and Management, 6(1), 2-10.

Behn, R. D. (1998). What right do Public Managers Have to Lead . Public Administration Review Vol $58 N^{\circ} 3,209-225$.

Corzo, F. L., Coronel, M., Arlet, J., Suca, F. R., \& Velásquez, M. J. (2018). Causas de la rotación laboral en las empresas del sector asegurador de Cusco.

DeVellis, R. (2003). Scale development, theory and application. Chapel Hill, NC: Sage.

Eitzen, S., \& Yetman, N. (1972). Managerial Change, Longevity and Organizational Effectiveness. Administrative Science Quarterly Vol $17 N^{\circ} 1,110-116$.

Fairholm, M. R. (2004). Different Perspectives on the Practice of Leadership. Public Administration Review Vol $64 N^{\circ}$ 5, 577-590.

Hambrick, D., \& Fukutomi, G. (1991). The Seasons of a CEO’s Tenure. Academy of Management Review Vol $16 N^{\circ} 4,719-742$.

Hayes, A. F. (2017). Introduction to mediation, moderation, and conditional process analysis: A regression-based approach. . Guilford publications.

Ley Orgánica de Servicio Público, Registro Oficial Suplemento 294 de 06-oct.-2010. (2010). Quito.

Manosalvas, C., Manosalvas, L., \& Quintero, J. (2015). El clima organizacional y la satisfacción laboral: un análisis cuantitativo riguroso de su relación. AD-minister, (26), 5-15.

Plan Nacional de Desarrollo, 2.-2. (2017). Plan Nacional de Desarrollo 2017-2021. Toda una vida.

Reglamento General a la Ley Orgánica del Servicio Público, Registro Oficial Suplemento 418 de 01-abr.-2011. (2011). Quito.

Tariq, M., Ramzan, M., \& Riaz, A. (2013). The impact of employee turnover on the efficiency of the organization. Interdisciplinary Journal of Contemporary Research in Business 4(9), 700-711. 
Vermeeren, B., Kuipers, B., \& Steijn, B. (2014). Does Leadership Style Make a Difference? Linking HRM, Job Satisfaction and Organizational Performance. Review of Public Personnel Administration Vol $34 N^{\circ} 2,174-195$.

Ysa, T., \& Salvador, S. (2015). Liderazgo y dirección pública: el ciclo de vida del cargo directivo. Revista del CLAD Reforma y Democracia, (62), 39-76. 